Review

\title{
Experimental DNA- or RNA-Directed Therapies for Trinucleotide Repeat Disease
}

Inês Lopes Cardoso ${ }^{*}$

PhD in Biotechnology by Biotechnology Superior School of Portuguese Catholic University. Associate Professor at Fernando Pessoa University, Porto, Portugal; E-Mail: mic@ufp.edu.pt

* Correspondence: Inês Lopes Cardoso; E-Mail: mic@ufp.edu.pt

Academic Editor: Khue Vu Nguyen

Special Issue: Treatment of Genetic Disease

OBM Genetics

2018 , volume 2 , issue 3

doi:10.21926/obm.genet.1803025
Received: April 17, 2018

Accepted: July 24, 2018

Published: July 27, 2018

\begin{abstract}
:
Some repeats of three or more nucleotides in tandem, which are present in a gene or in its vicinity, tend to increase in number and for this reason are called dynamic mutations. These triplet repeats are unstable and can expand from one generation to the next. According to the expansion size, an unaffected individual can carry a pre-mutation that will expand through generations leading to the development of triplet repeat expansion diseases. The increase in the number of repeats over time leads to earlier development and increased severity of symptoms in affected individuals in successive generations. Although there is still no treatment for this type of disease, several strategies are under investigation. Here, we describe treatment approaches for triplet repeat expansion diseases that have been developed over recent years, using DNA or RNA molecules as targets. Some of these strategies have the potential for future use in gene therapy for trinucleotide repeat disorders.
\end{abstract}

\section{Keywords}

Treatment of trinucleotide repeat diseases; RNA interference; antisense oligonucleotides; experimental therapies; Myotonic Dystrophy; Huntington disease the conditions of the Creative Commons by Attribution License, which permits unrestricted use, distribution, and reproduction in any medium or format, provided the original work is correctly cited. 


\section{Introduction}

All eukaryotic and prokaryotic genomes contain a certain number of repetitive sequences that can vary in length and repetitive levels similar to long repeats and satellite DNA [1-4]. Overall, 30\% of the human genome consists of repetitive DNA sequences [5].

The presence of trinucleotide repeats (microsatellites), also known as tandem repeat sequences, that are responsible for the development of diseases was identified as a novel type of mutation in the early 1990s [6]. This type of repetitive sequence is susceptible a high frequency of mutations in all genomes $[2,7]$.

Diverse hereditary neurological disorders are caused by expansion of unstable triplet repeats [8]. Trinucleotide repeat expansions have been identified as the cause of approximately 20 neurologic and neuromuscular diseases, although the mechanisms underlying their development remain to be elucidated $[5,9]$. Examples of this type of disease include myotonic dystrophy (DM) Huntington disease (HD), fragile $X$ syndrome, and many others resulting from the accumulation of triplet repeats (CTG-CAG, CGG-CCG or GAA-TTC) in corresponding genes $[9,10]$. Symptoms start to develop in carriers of the repeat as soon as a sufficient number of triplet copies are acquired. This number increases in successive generations due to somatic instability of the expanded sequence, leading to the unusual pattern of genetic anticipation, a phenomenon in which each generation shows earlier onset of the disease together with increasing severity of the symptoms $[9,10]$.

Despite great efforts in the last decade, there is still no cure for these disorders [6]; however, several treatment strategies are under development. Here, we discuss the most recently studied treatment approaches directed against nucleic acids, some of which have potential applications in future gene therapy of trinucleotide repeat expansion disorders.

\section{Materials and Methods}

This review was conducted through searches of scientific publications, review papers, books and theses available in the PubMed, B-On, SciELO, and Science Direct databases, as well as the libraries of O'Porto Medicine Faculty (FMUP) and Fernando Pessoa University. Searches were performed using the following keywords were: "treatment of trinucleotide repeat diseases", "diseases of trinucleotide repeats", "neurodegenerative disorders", "spinocerebellar ataxia", "Huntington disease", "fragile X disease", "myotonic dystrophy", "Friedrich ataxia", "fragile Xassociated ataxia".

\section{Experimental Therapies Directed Against Triplet Repeats}

Development of selective therapeutic strategies is based on differences between normal and mutant transcripts in terms of repeat sequence length and assumed secondary structures [6].

The most promising approaches for possible treatments are aimed at lowering mutant protein synthesis by targeting DNA or RNA transcripts in cases of diseases where there is a gain-offunction. 
On the other hand, other strategies aimed at increasing expression of the wild-type gene by targeting DNA are under development for the treatment of genetic diseases resulting from decreased gene expression.

The treatment approaches discussed here are divided in accordance with the target nucleic acid: RNA transcript or DNA.

\subsection{RNA-Targeting Strategies}

RNA-targeting has been developed using three main strategies directed against triplet RNA repeats: RNA interference (RNAi), antisense oligonucleotides (ASOs) or the use of splicing inhibitors.

RNA Interference (RNAi). RNA interference (RNAi) is based on degradation of mutant transcripts since these RNA molecules bind to mRNA in the cytoplasm, leading to its degradation by the RNase enzyme, argonaute 2 [11].

These antisense reagents, together with antisense oligonucleotides, are known as "cutters" since they attach to complementary targets and induce their cleavage.

In 2015, Bisset et al. [12] proposed this strategy as a promising therapeutic approach for genetic disorders involving gain-of-function. These researchers designed miRNA-based RNAi hairpins to target the mutant CUG expansion mRNA in a mouse model of myotonic dystrophy type 1 (DM1). As a route to systemic gene therapy in mice, the RNAi expression cassettes were delivered by intravenous injection of recombinant adeno-associated viral vectors. A significant reduction in disease pathology in the muscles of treated mice was observed, together with reduced mutant mRNA levels and a decrease in myonuclear foci containing the mutant mRNA. It was concluded that RNAi has potential as a long-term therapy for DM1 and other dominant muscular dystrophies [12].

This methodology has also been used in studies of the treatment of Huntington disease. Harper et al. [13] demonstrated that RNAi directed against the mutant human htt gene, reduced huntingtin expression at both the mRNA and protein levels in cell cultures and HD mouse brain. Furthermore, delivery of adeno-associated virus constructs containing short hairpin RNAs that reduced $h t t$ gene expression resulted in improvements in the neuropathological changes associated with HD in a mouse model [13].

Franich et al. [14] used RNAi to target mutant $h t t$ gene expression mediated by virus vector delivery in HD transgenic mouse models. This strategy was shown to mediate a dramatic knockdown in mutant HD gene expression, preventing striatal neurodegeneration and concomitant motor behavioral impairment [14].

In a study of the safety of RNAi treatment in primates, McBride et al. [15] demonstrated that a $45 \%$ reduction in rhesus $h t t$ gene expression did not induce motor deficits or neuronal degeneration. This study suggests that partial suppression of wild-type htt expression is welltolerated in the primate and further supports the use of RNAi as a therapy for HD [15].

Miniarikova et al. [16] evaluated two RNAi-based approaches for the treatment of HD. They observed total silencing of wild-type and mutant huntingtin (HTT) protein following RNAimediated targeting of exon 1 of the $h t t$ gene. These researchers also used an allele-specific silencing strategy based on targeting the heterozygous single nucleotide polymorphism rs362331 in exon 50 or rs362307 in exon 67, which are linked to mutant HTT [16]. They observed strong 
allele-selective silencing of mutant HTT when targeting rs362331 both in vitro and in vivo [16]. This study showed that a single treatment provides a sustained reduction in HTT expression.

RNAi has been proposed as a strategy for gene therapy of genetic disorders. As a good example of this approach, Keiser et al. [17] reported that spinocerebellar ataxia type 1 could be reversed by RNAi therapy. These researchers had previously shown that partial suppression of mutant ataxin-1 gene expression following delivery of a single dose of virus could prevent the development of disease symptoms in a transgenic mouse model and a knock-in mouse model of the disease [18]. Their subsequent investigations then showed that RNAi not only prevented, but also reversed disease development when delivered after the onset of symptoms. In this study, a recombinant adeno-associated virus expressing an artificial miRNA targeting human ATXN1 mRNA was delivered at multiple doses in a mouse model of spinocerebellar ataxia type 1 , before or after symptom onset [17].

Antisense Oligonucleotides (ASOs). Antisense oligonucleotides (ASOs) are complementary to mutant mRNAs, and thus hybridize with them, catalysing degradation of these transcripts by $\mathrm{RNase} \mathrm{H}$ and consequently, leading to a reduction in coded protein levels [6].

Wheeler et al. $[19,20]$ observed that DM1 nuclear expansions are highly sensitive to antisense silencing. A transgenic mouse model of DM1 expressing human skeletal actin transcripts with the expansion (CUG) 250 inserted in the $3^{\prime}$-UTR was used to show that it is possible to reverse changes observed in myotonic dystrophy. This was achieved by administration of a morpholino ASO, CAG25, which binds to $C_{\text {exp }}$ RNA and blocks its interaction with $C_{\text {exp }}$-binding proteins [19]. The morpholino ASO was loaded into muscle fibers by intramuscular injection followed by in vivo electroporation. The muscular tissue was then examined 1-3 weeks later by fluorescence in situ hybridization using probes that hybridize to the CUG repeat or to sequences flanking the repeat. Injection of CAG25 caused a marked reduction in nuclear foci and a redistribution of

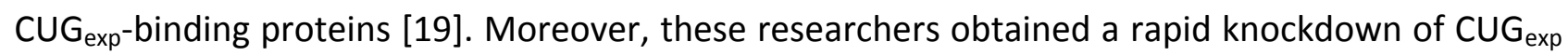
RNA in skeletal muscle in the DM1 mouse model by systemic administration of ASOs [20].

In the same way, Mulders et al. [21] reported the use of ASOs in a mouse DM1 cell model and DM1 patient cells. Cells were transfected with ASOs complexed with polyethyleneimine. A (CAG) 7 ASO silenced RNA expression of mutant myotonic dystrophy protein kinase and reduced the number of ribonuclear aggregates. The study also showed that direct administration of this ASO into the muscle of DM1 model mice caused a significant reduction in the level of toxic RNA [21].

Other researchers used ASOs containing modified nucleic acid residues, known as "gapmers", to induce RNase $\mathrm{H}$-mediated degradation of DM1 pathogenic transcripts [22]. Using RT-PCR to quantify the mutant transcript, this study showed selective knockdown of expanded CUG transcripts and consequent disruption of RNA foci both in cell culture and DM1 mouse models [22]. Furthermore, these researchers concluded that the combination of gapmers and morpholino ASOs has the potential to enhance the knockdown effect [22].

ASOs were also tested in the treatment of HD. Kordasiewicz et al. [23] observed a delay in disease progression following transient infusion of ASOs into the cerebrospinal fluid in a symptomatic HD mouse model. They also observed a sustained reversal of the disease phenotype that persisted longer than the huntingtin knockdown. Reduction of wild-type huntingtin, along with mutant huntingtin, produced the same sustained disease reversal. ASO infusion into nonhuman primates had a similar effect, with an $80 \%$ reduction in huntingtin transcript levels observed using this strategy [23]. 
Moreover, using an HD mouse model, Datson et al. [24] observed a reduction (15\%-60\%) in soluble and aggregated mutant HTT protein in the brains of mice following infusions of (CUG $)_{7}$ ASOs [24].

ASOs were also tested in the treatment of the neurodegenerative disorder spinocerebellar ataxia type-3. Evers et al. [25] attempted to remove the toxic polyglutamine repeat from the atazin-3 protein through antisense oligonucleotide-mediated exon skipping. This strategy produced a reduction in mutant ataxin-3 toxicity, while maintaining important functions of the wild-type protein. In vitro studies showed that exon skipping did not negatively impact the ubiquitin-binding capacity of ataxin-3 and no toxic properties of the novel truncated ataxin-3 protein were detected in the in vivo tests [25]. These researchers used this strategy for skipping of exon 8 and 9, resulting in the removal of a central region of 88 amino acids in the ataxin-3 protein that contains several predicted cleavage sites and two ubiquitin-interacting motifs. In vitro studies showed that the truncated ataxin-3 protein did not show cellular toxicity but was unable to bind poly-ubiquitin chains, which may interfere with its normal de-ubiquitinating function. Therefore, these researchers concluded that skipping of exon 8 and 9 is not a viable therapeutic option for this disorder [26]. However, exon 10 skipping led to the formation of a truncated ataxin-3 protein lacking the toxic polyglutamine expansion and retaining its ubiquitin-binding and cleavage function. These data suggested that exon 10 skipping is a promising therapeutic approach for spinocerebellar ataxia type 3 [27].

Splicing Inhibitors. Splicing inhibitors have been used in the treatment of spinal muscular atrophy, which results from deficiency of a protein required for the survival of motor neurons. This genetic disorder is caused by mutation or deletion of the survival motor neuron 1 (SMN1) gene. In the absence of this protein, neurons gradually die and patient's muscles waste away, leading to death. Naryshkin et al. [28] discovered small-molecule drugs that increase synthesis of the missing protein by inhibiting the alternative splicing that leads to the generation of the truncated SMN protein and neuromuscular degeneration [28]. In mouse models of severe spinal muscular atrophy, administration of these compounds produced a marked improvement in muscle mass, motor function and survival [28]. These splicing modifiers may have therapeutic potential for patients with spinal muscular atrophy.

Furamidine, a RNA CAG Binder. In Huntington disease, the mutant CAG-repeat RNA codes for neurotoxic polyglutamine proteins and can lead to a toxic gain-of-function by recruitment of RNAbinding proteins. The link between these molecules induces aberrant HTT protein translation. Matthes et al. [29] identified a set of CAG repeat binders, one of which, furamidine, reduced the binding of huntingtin mRNA to several RNA-binding proteins in vitro [29]. Moreover, furamidine also decreased HTT protein levels in an HD cell line model [29]. However, this ligand, which can also bind to AU RNAs, CUG RNAs or even the DNA minor groove (AT), seems to bind the CAGrepeat RNA in an unspecific manner and only when present in high concentrations. Thus, due to the high risk of off-target effects, furamidine is not a good candidate as an anti-HD drug. Nevertheless, this study shows that small molecules that mask RNA-protein interactions may be active against the mutant HTT protein [29]. 


\subsection{DNA Targeting Strategies}

In trinucleotide repeat diseases where there is a gain-of-function, targeting DNA to reduce protein expression represents a strategy for gene therapy. Several approaches are currently being tested, including the use of zinc finger proteins, clustered regularly interspaced short palindromic repeats (CRISPR)/CRISPR-associated 9 (Cas9), and transcription activator-like effector nucleases (TALENs) as well as peptide nucleic acid (PNA)-peptide conjugates and locked nucleic acid (LNA) oligomers.

The main advantage of the use of zinc finger proteins, TALENs or CRISPR/Cas9 is that these strategies allow the introduction of a double-stranded break at specific points in the DNA and thus, induce homology-directed repair [30]. These therapies have been tested for the insertion of a functional copy of an entire gene in a precisely targeted region. They can also be used to correct pathogenic mutations in situ [30].

In other triplet repeat diseases, such as Friedreich's ataxia or spinal muscular dystrophy, the expanded repeat leads to a reduction in gene expression. In addition to the already described RNA-targeting strategies that can lead to increased gene expression, other methods have been developed to target DNA directly (for example ASOs), induce pharmacological modulation or inhibit histone deacetylase (HDAC).

Zinc Finger Proteins. Zinc finger proteins can be targeted to specific DNA sequences and form a structural motif able to bind DNA, resulting in a reduction in protein synthesis.

This strategy has been used to reduce levels of mutant huntingtin protein in HD animal models. For example, Garriga-Canut et al. [31] used long artificial zinc finger protein chains, designed to bind longer CAG-repeats more strongly than shorter repeats, in an HD mouse model. Based on the ability to recognize both poly-CAG and its complementary DNA strand, the designed zinc finger proteins recognized and bound to poly-5'-GC (A/T)-3'. These chains mediated preferential repression of target genes with longer CAG-repeats, when compared with shorter repeats following transient transfection and stable expression in various HD model cell lines. The stable expression of this zinc finger protein in an HD cell line model reduced chromosomal expression of the mutant gene at both the protein and mRNA levels (95\% and 78\% reduction, respectively) [31]. Wild-type gene expression was not affected by this strategy. Similar effects were observed following adeno-associated virus delivery in an HD mouse model in vivo.

Similarly, Agustin-Parón et al. [32] designed a gene therapy strategy using a zinc finger transcription repressor that binds to expanded DNA CAG-repeats in the huntingtin gene. This study showed repression reaching $77 \%$ of the mutant huntingtin gene expression in whole brains of HD model mice [32]. A 48\% repression was still observed 12 weeks after treatment and a $23 \%$ repression after 24 weeks, demonstrating that long-term effects can be achieved using this approach. However, since these proteins lead to the formation of non-native molecules, there is a risk increased immunogenicity and developing immune reactions [32].

Moreover, Kim and Kim [33] reported cytotoxicity, off-target effects and relatively low efficiency using zinc finger proteins [33].

Clustered Regularly Interspaced Short Palindromic Repeats (CRISPR)/CRISPR-Associated 9 (Cas9). In the bacterial immune system, the clustered regularly interspaced short palindromic repeats (CRISPR)/CRISPR-associated 9 (Cas9) gene-editing system can be used to introduce 
double-stranded breaks in foreign DNA with high efficiency, low cytotoxicity, and relatively few off-target effects [34].

The Cas9 protein cuts target DNA, and the targeted sequence is repaired by gene-editing processes involving the non-homologous end-joining (NHEJ) and homology-directed repair (HDR) mechanisms. NHEJ results in the addition or deletion of nucleotides, whereas, HDR editing results in full DNA sequence restoration and can only be used when a homologous DNA sequence is present [35].

In HDR, targeted introduction of a donor sequence into the genome at the position of a CRISPR/Cas9-mediated DNA break is performed using genome-engineered constructs carrying homologous sequences [35].

In a recent study, this novel strategy was shown to induce the recovery of a normal phenotype in myogenic cells derived from fibroblasts of patients with myotonic dystrophy type 1 [36]. With the aim of eliminating the toxic mutant repeats, CRISPR/Cas9 facilitated removal of the repeat expansions, therefore, preventing nuclear foci formation and splicing alterations [36]. Compared with previously described strategies, this study demonstrated the advantage of this approach in that affected cells permanently reverted to the normal phenotype; however, off-target activity was observed [36]. Subsequent studies focused on improvement of the targeting specificity and adjustable expression of Cas9 nuclease, which are critical for therapeutic application of this strategy, since genome editing leads to permanent modifications.

Pinto et al. [37] tested the hypothesis that deactivation of the Cas9 enzyme impedes transcription across expanded microsatellites in DM1 cells. Systemic delivery of dCas9/gRNA by adeno-associated virus led to a reduction in mutant RNA and decreased myotonia [37]. These results indicate that transcription of microsatellite repeat-containing RNAs is more sensitive to the action of Cas9 than transcription of other RNAs, making it a potential strategy for therapeutic intervention.

Permanent inactivation of the Huntington disease mutation in fibroblasts of a patient was also achieved by Shin et al. using this approach [38]. In this study, the use of haplotype-specific CRISPR/Cas9 was demonstrated to inactivate the mutant HD allele. Two CRISPR/Cas9 guide RNAs that depend on protospacer-adjacent motif sites resulting from single nucleotide polymorphism alleles on the mutant chromosome were used to selectively excise a region of DNA (approximately $44 \mathrm{~kb}$ ) spanning the promoter region, transcription start site, and the CAG expansion mutation of the mutant $h t t$ gene, resulting in complete inactivation of the mutant allele without changes in the wild-type allele [38]. This prevented the formation of mutant HTT mRNA and protein, unequivocally indicating permanent mutant allele-specific inactivation of the HD mutant allele [38]. Potentially, this strategy could be used as gene therapy for disorders with diverse disease haplotypes, supporting precision medicine by inactivation of gain-of-function mutations.

Moreover, a recent study described the development of a programmable CRISPR system capable of specifically visualizing and eliminating toxic RNAs resulting from microsatellite repeat expansions present in DNA [39]. Researchers observed reversal of hallmark features of disease, including elimination of RNA foci, in the cells of patients of affected by each of the studied diseases (DM1, DM2, C9-ALS, polyglutamine diseases) [39].

Several studies compared the mutagenic rates of CRISPR/Cas9, zinc finger proteins and TALENs. Kim and Kim [33] observed similar mutation rates for all methods, while Auer and Del Bene [35] showed CRISPR/Cas9 mutation rates ranging between $42 \%$ and $100 \%$ compared with a mutation 
rate of $33 \%$ for TALENs [35]. In another study, mutation rates of $11 \%$ for TALENs and $76 \%$ for CRISPR-Cas9, were recorded for the same gene [40].

In the same way, He et al. [41] observed more efficient targeted genomic deletions together with more precise re-joining of breakpoint junctions induced by CRISPR/Cas9 compared with the use of TALENs. In comparisons of the ability to edit an integrated gene, CRISPR/Cas9 was found to be superior for targeted genomic deletions, while TALENs outperformed CRISPR/Cas9 for stimulation of concurrent HDR at proximal sites within a single gene [41].

Kolli et al. [42] reported a relatively low rate of HDR using CRISPR/Cas9 and other studies suggest that the rate of HDR is dependent on gRNA concentration and Cas9 protein availability [43, 44].

A recent study showed similar behavior of heterodimeric zinc finger proteins and CRISPR/Cas9 for homology-directed gene knock-in strategies (88\% and 83\%, respectively, of the donors inserted in the target locus), whereas homodimeric zinc finger proteins showed only 45\% on-target insertions [45].

Although CRISPR/Cas9 has enormous potential for gene therapy and genome editing applications, some challenges need to be overcome before this approach can be used for the treatment of human diseases. One of the most important challenges, is the reduction of off-target effects. Since the gRNA of the CRISPR/Cas9 targeting system is only 17-24 bp in length, there is a high chance that the selected sequence exists elsewhere besides the target regions. Moreover, sequences with small changes to the target gRNA sequence might exist in the genome and can cause mis-targeting. However, several studies suggest that Cas 9 can withstand mismatches, and is dependent on the distribution and number of mismatched nucleotides [46-48]. Kim and Kim [33] reported that off-target effects of CRISPR/Cas9 system can be reduced by altering the Cas 9 enzyme to act as a nickase, capable of cleaving only one target DNA strand [33].

Transcription Activator-Like Effector Nucleases (TALENs). Like zinc finger nucleases and CRISPR)/CRISPR-Cas9, transcription activator-like effector nucleases (TALENs) are engineered nucleases that have been widely used to generate double-strand DNA breaks (DSBs) to increase the efficiency of standard homologous recombination. Mosbach et al. [49] showed that a TALENinduced double-stranded break was very efficient at contracting expanded CTG repeats in yeast. This study showed that, following the TALEN-induced double-strand break, single-strand annealing occurred between both sides of the repeat tract, leading to repeat contraction [49]. These results implicate TALENs as a promising strategy for gene therapy of trinucleotide repeat disorders; however, although TALENs have a higher efficiency than zinc finger proteins, it requires huge resources, and delivery into cells is often a challenge [33, 34].

Peptide Nucleic Acid (PNA)-Peptide Conjugates and Locked Nucleic Acid (LNA) Oligomers. An important issue in therapy directed against triplet repeats is the need for gene and allele selectivity. Although numerous mRNAs in the human transcriptome contain triplet repeats, the specific inhibition of mutant gene expression by targeting certain repeat regions is a promising therapy strategy for these types of diseases [6].

Agents have been developed to distinguish between RNA hairpin structures formed by wildtype and mutant RNAs based on differences in length and consequently, in stability.

Hu et al. [50] used peptide nucleic acid (PNA)-peptide conjugates and locked nucleic acid (LNA) oligomers for specific reduction of mutant huntingtin gene expression [50]. PNAs, which are a class of DNA/RNA mimic with an uncharged amide backbone, are advantageous as agents for 
binding complementary RNA sequences due to the lack of charge-charge repulsion of RNA/DNA or RNA/RNA duplexes. This results in an increase in the affinity of PNA hybridization and recognition of target sequences. PNAs can be delivered to live cells and are resistant to proteases and nucleases, which makes them promising therapeutic agents. PNA oligomers hybridize to DNA and RNA in a sequence-specific manner. Kiliszek et al. [51] demonstrated the high sequence-specificity of PNAs in studies of the crystal structures of RNA-PNA duplexes and a PNA-PNA duplex containing mismatches. Using this technique, only fully complementary RNA-PNA duplexes could be obtained, while co-crystallization trials of mismatching RNA with PNA resulted only in crystals of mismatched PNA-PNA [51]. This sequence selectivity is a desirable property for antisense therapeutic strategies against trinucleotide repeat expansion diseases since it will allow inhibition of mutant gene expression without interfering with the wild-type gene.

On the other hand, LNAs are RNA analogs containing a methylene bridge between the 2'oxygen and 4'-carbon of the ribose. This bridge reduces the conformational flexibility of ribose and confers outstanding affinity in complementary hybridization. Hu et al. [50] observed that LNAs, as well as previously described PNAs, can confer potent allele-selective inhibition of mutant huntingtin gene expression [50]. In the same way, Rué et al. [52] observed that a LNA-modified antisense oligonucleotide complementary to the CAG-repeat (LNA-CTG) preferentially bound to mutant $h t t$ gene without affecting wild-type HTT mRNA or protein levels. Furthermore, these researchers observed that this strategy promotes neuroprotection in a mouse model of HD by blocking the detrimental activity of CAG-repeats in the htt gene [52].

Antisense Oligonucleotides (ASOs). ASOs have also been used to increase gene expression in cell lines derived from patients with Friedreich's ataxia and spinal muscular dystrophy $[53,54]$. The proposed mechanism by which ASOs increase gene expression involves the binding of these compounds to the expanded repeat and blockade of the expanded RNA, which inhibits recognition of DNA, prevents R-loop formation and allows more gene expression.

A study by Li et al. [53] demonstrated that ASOs and dsRNAs can activate frataxin protein expression in Friedreich's ataxia patient-derived cell lines that possess varied numbers of GAA repeats. Increased frataxin protein expression was achieved by ASOs incorporating diverse chemical modifications [53].

Using the same strategy, Corey et al. [54] showed the nusinersen (Spinraza), a recently approved antisense oligonucleotide drug, can be used for the treatment of spinal muscular atrophy [54].

Pharmacological Modulation during Somatic Cell Reprogramming. Another recently evaluated strategy was based on the hypothesis that the epigenetic modifications close to GAA repeats present in Friedreich's ataxia can be reversed by pharmacological modulation during somatic cell reprogramming [55]. In this study, researchers induced the generation of pluripotent stem cells by reprogramming Friedreich's ataxia fibroblasts in the presence of various small molecules that target DNA methylation as well as histone acetylation and methylation. Treatment of these stem cells with two compounds (sodium butyrate and Parnate [tranylcypromine]), led to increased expression of the frataxin gene, which persisted for several passages [55]. However, they observed that prolonged culture of those epigenetically modified cells resulted in progressive expansion of triplet repeats and a consequent decrease in gene expression.

Histone Deacetylase (HDAC) Inhibitors. Evidence indicates that transcriptional dysregulation is a contributor to the molecular pathogenesis of Huntington disease. For this reason, histone 
deacetylase (HDAC) inhibitors have been tested in the treatment of Huntington disease, with the aim of correcting transcriptional dysregulation through chromatin modification. These inhibitors prevent removal of acetyl groups from histones. As a result, DNA assumes a more relaxed structure, allowing access of transcription factors to DNA and consequently, activating gene transcription.

Hockly et al. [56] explored the therapeutic potential of HDAC inhibitors by conducting preclinical trials with a potent HDAC inhibitor, suberoylanilide hydroxamic acid (SAHA), in a mouse model of HD. These researchers showed that this molecule crosses the blood-brain barrier and increases histone acetylation in the brain. Furthermore, this treatment dramatically improved motor impairment of HD mice [38]. Moreover, Mielcarek et al. [57] demonstrated that SAHA also promotes the degradation of several classes of HDACs at the protein level in various cancer cell lines and in the cortex and brain stem in mouse models.

\section{Conclusion}

Trinucleotide repeat expansions are responsible for the development of several genetic disorders. The instability of these repeats leads to their expansion and to the accumulation of increasing numbers of triplets from one generation to the next. This results in the phenomenon of anticipation, which is characteristic of triplet diseases, where development of symptoms occurs earlier in each generation.

To date, there is no cure for this type of disorder, although several possible treatment strategies are under investigation. The goal of most available treatments is to relieve symptoms and provide patients with a better life quality. However, the possibility of a cure for affected patients should be explored. Therefore, several approaches directed against DNA or RNA are under development based on the potential of these approaches to achieve prolonged, or even permanent, reversal of symptomatology.

Some of the approaches described interfere not only with the mutant gene or mRNA, but also with the wild-type, and for this reason might lead to dysregulation of gene expression. To avoid further adverse effects, it is essential that the treatment strategy is directed specifically to the mutant nucleic acids.

Moreover, several of the discussed strategies allow the removal of the repeat expansion, leading to total recovery of the wild-type phenotype. These treatment approaches offer the potential for the establishment of a cure for trinucleotide repeat diseases.

\section{Author Contributions}

Inês Lopes Cardoso did all the research work for this study.

\section{Competing Interests}

The authors have declared that no competing interests exist. 


\section{References}

1. Boeva V, Regnier M, Papatsenko D, Makeev V. Short fuzzy tandem repeats in genomic sequences, identification, and possible role in regulation of gene expression. Bioinformatics. 2006; 22: 676-684.

2. Huntley MA, Golding GB. Neurological proteins are not enriched for repetitive sequences. Genetics. 2004; 166: 1141-1154.

3. Subramanian S, Madgula V, George R, Mishra RK, Pandit MW, Kumar CS, et al. Triplet repeats in human genome: distribution and their association with genes and other genomic regions. Bioinformatics. 2003; 19: 549-552.

4. McConnell R, Middlemist S, Scala C, Strassmann JE, Queller DC. An unusually low microsatellite mutation rate in Dictyostelium discoideum, an organism with unusually abundant microsatellites. Genetics. 2007; 177(3):1499-1507.

5. McMurray CT. Mechanisms of trinucleotide repeat instability during human development. Nat Rev Genet. 2010; 11: 786-799.

6. Krzyzosiak WJ, Sobczak K, Wojciechowska M, Fiszer A, Mykowska A, Kozlowski P. Triplet repeat RNA structure and its role as pathogenic agent and therapeutic target. Nucleic Acids Res. 2012; 40: 11-26.

7. Sobczak K, Michlewski G, de Mezer M, Kierzek E, Krol J, Olejniczak M, et al. Structural diversity of triplet repeat RNAs. J Biol Chem. 2010; 285: 12755-12764.

8. Jakupciak JP, Wells RD. Genetic instabilities in (CTG.CAG) repeats occur by recombination. J Biol Chem. 1999; 274: 23468-23479.

9. Jung J, van Jaarsveld MT, Shieh SY, Xu K, Bonini NM. Defining genetic factors that modulate intergenerational CAG repeat instability in Drosophila melanogaster. Genetics. 2011; 187: 6171.

10. Jakupciak JP, Wells RD. Genetic instabilities of triplet repeat sequences by recombination. IUBMB Life. 2000; 50: 355-359.

11. Hutvagner G, Simard MJ. Argonaute proteins: key players in RNA silencing. Nat Rev Mol Cell Biol. 2008; 9: 22-32.

12. Bisset DR, Stepniak-Konieczna EA, Zavaljevski M, Wei J, Carter GT, Weiss MD, et al. Therapeutic impact of systemic AAV-mediated RNA interference in a mouse model of myotonic dystrophy. Human Mol Genet. 2015; 24: 4971-4983.

13. Harper SQ, Staber PD, He X, Eliason SL, Martins IH, Mao Q, et al. RNA interference improves motor and neuropathological abnormalities in a Huntington's disease mouse model. Proc Natl Acad Sci USA. 2005; 102: 5820-5825.

14. Franich NR, Fitzsimons HL, Fong DM, Klugmann M, During MJ, Young D. AAV vector-mediated RNAi of mutant huntingtin expression is neuroprotective in a novel genetic rat model of Huntington's disease. Mol Ther. 2008; 16: 947-956.

15. McBride JL, Pitzer MR, Boudreau RL, Dufour B, Hobbs T, Ojeda SR, et al. Preclinical safety of RNAi-mediated HTT suppression in the rhesus macaque as a potential therapy for Huntington's disease. Mol Ther. 2011; 19: 2152-2162.

16. Miniarikova J, Zanella I, Huseinovic A, van der Zon T, Hanemaaijer E, Martier R, et al. Design, characterization, and lead selection of therapeutic miRNAs targeting huntingtin for development of gene therapy for Huntington's disease. Mol Ther Nucleic Acids. 2016; 5: e297. 
17. Keiser MS, Monteys AM, Corbau R, Gonzalez-Alegre P, Davidson BL. RNAi prevents and reverses phenotypes induced by mutant human ataxin-1. Ann Neurol. 2016; 80: 754-765.

18. Keiser MS, Kordower JH, Gonzalez-Alegre P, Davidson BL. Broad distribution of ataxin 1 silencing in rhesus cerebella for spinocerebellar ataxia type 1 therapy. Brain. 2015; 138: 35553566.

19. Wheeler TM, Sobczak K, Lueck JD, Osborne RJ, Lin X, Dirksen RT, Thornton CA. Reversal of RNA dominance by displacement of protein sequestered on triplet repeat RNA. Science. 2009; 325: 336-339.

20. Wheeler TM, Leger AJ, Pandey SK, Macleod AR, Nakamori M, Cheng SH, et al. Targeting nuclear RNA for in vivo correction of myotonic dystrophy. Nature. 2012; 488: 111-115.

21. Mulders SA, van den Broek WJ, Wheeler TM, Croes HJ, van Kuik-Romeijn P, de Kimpe SJ, et al. Triplet-repeat oligonucleotide-mediated reversal of RNA toxicity in myotonic dystrophy. P Natl Acad Sci USA. 2009; 106: 13915-13920.

22. Lee JE, Bennett CF, Cooper TA. RNase H-mediated degradation of toxic RNA in myotonic dystrophy type 1. P Natl Acad Sci USA. 2012; 109: 4221-6.

23. Kordasiewicz HB, Stanek LM, Wancewicz EV, Mazur C, McAlonis MM, Pytel KA, et al. Sustained therapeutic reversal of Huntington's disease by transient repression of huntingtin synthesis. Neuron. 2012; 74: 1031-44.

24. Datson NA, González-Barriga A, Kourkouta E, Weij R, van de Giessen J, Mulders S, et al. The expanded CAG repeat in the huntingtin gene as target for therapeutic RNA modulation throughout the HD mouse brain. PLOS ONE. 2017; 12: e0171127.

25. Evers MM, Tran HD, Zalachoras I, Pepers BA, Meijer OC, den Dunnen JT, et al. Ataxin-3 protein modification as a treatment strategy for spinocerebellar ataxia type 3: removal of the CAG containing exon. Neurobiol. 2013; 58: 49-56.

26. Toonen L, Schmidt I, Luijsterburg MS, van Attikum H, van Roon-Mom WM. Antisense oligonucleotide-mediated exon skipping as a strategy to reduce proteolytic cleavage of ataxin3. Sci Rep. 2016; 6: 35200.

27. Toonen LJA, Rigo F, van Attikum H, van Roon-Mom WMC. Antisense oligonucleotide-mediated removal of the polyglutamine repeat in spinocerebellar ataxia type 3 mice. Mol Ther Nucleic Acids. 2017; 8: 232-242.

28. Naryshkin NA, Weetall M, Dakka A, Narasimhan J, Zhao X, Feng Z, et al. Motor neuron disease. SMN2 splicing modifiers improve motor function and longevity in mice with spinal muscular atrophy. Science. 2014; 345: 688-93.

29. Matthes F, Massari S, Bochicchio A, Schorpp K, Schilling J, Weber S, et al. Reducing mutant Huntingtin protein expression in living cells by a newly identified RNA CAG binder. ACS Chem Neurosci. 2018; doi: 10.1021/acschemneuro.8b00027.

30. Ott de Bruin LM, Volpi S, Musunuru K. Novel genome-editing tools to model and correct primary immunodeficiencies. Front Immuno. 2015; 6: 250.

31. Garriga-Canut M, Agustín-Pavón C, Herrmann F, Sánchez A, Dierssen M, Fillat C, et al. Synthetic zinc finger repressors reduce mutant huntingtin expression in the brain of R6/2 mice. P Natl Acad Sci USA. 2012; 109: E3136-3145.

32. Agustin-Pavón C, Mielcarek M, Garriga-Canut M, Isalan M. Deimmunization for gene therapy: host matching of synthetic zinc finger constructs enables long-term mutant huntingtin repression in mice. Mol Neurodegener. 2016; 11: 64. 
33. Kim H, Kim JS. A guide to genome engineering with programmable nucleases. Nat Rev Genet. 2014; 15: 321-334.

34. Carlson DF, Fahrenkrug SC, Hackett PB. Targeting DNA with fingers and TALENs. Mol Ther Nucleic Acids. 2012; 1: e3.

35. Auer TO, Del Bene F. CRISPR/Cas9 and TALEN-mediated knock-in approaches in zebrafish. Methods. 2014; 69: 142-50.

36. Provenzano C, Cappella M, Valaperta R, Cardani R, Meola G, Martelli F, et al. CRISPR/Cas9mediated deletion of CTG expansions recovers normal phenotype in myogenic cells derived from Myotonic Dystrophy 1 patients. Mol Ther Nucleic Acids. 2017; 9: 337-348.

37. Pinto BS, Saxena T, Oliveira R, Méndez-Gómez HR, Cleary JD, Denes LT, et al. Impeding transcription of expanded microsatellite repeats by deactivated Cas9. Mol Cell. 2017; 68: 479490.

38. Shin JW, Kim KH, Chao MJ, Atwal RS, Gillis T, MacDonald ME, et al. Permanent inactivation of Huntington's disease mutation by personalized allele-specific CRISPR/Cas9. Hum Mol Genet. 2016; 25: 4566-4576.

39. Batra R, Nelles DA, Pirie E, Blue SM, Marina RJ, Wang $H$, et al. Elimination of toxic microsatellite repeat expansion RNA by RNA-targeting Cas9. Cell. 2017; 170: 899-912.

40. Veres A, Gosis BS, Ding Q, Collins R, Ragavendran A, Brand H. et al. Low incidence of off-target mutations in individual CRISPR-Cas9 and TALEN targeted human stem cell clones detected by whole-genome sequencing. Cell stem cell. 2014; 15: 27-30.

41. He Z, Proudfoot C, Whitelaw BA, Lillico SG. Comparison of CRISP/Cas9 and TALENs on editing an integrated EGFP gene in the genome of HEK293FT cells. SpringerPlus. 2016; 5: 814.

42. Kolli N, Lu M, Maiti P, Rossignol J, Dunbar GL. Application of the gene editing tool, CRISP-Cas9, for treating neurodegenerative diseases. Neurochem Int. 2018; 112: 187-196.

43. Hwang WY, Fu Y, Reyon D, Maeder ML, Tsai SQ, Sander JD. et al. Efficient genome editing in zebrafish using a CRISPR Cas system. Nat Biotechnol. 2013; 31: 227-229.

44. Guo X, Zhang T, Hu Z, Zhang Y, Shi Z, Wang Q. et al. Efficient RNA/Cas9-mediated genome editing in Xenopus tropicalis. Development. 2014; 141: 707-714.

45. Gutierrez-Guerrero A, Sanchez-Hernandez S, Galvani G, Pinedo-Gomez J, Martin-Guerra R, Sanchez-Gilabert $A$. et al. Comparison of zinc finger nucleases versus CRISPR-specific nucleases for genome editing of the Wiskott-Aldrich syndrome locus. Hum Gene Ther. 2018; 29: 366-380.

46. Hsu PD, Lander ES, Zhang F. Development and applications of CRISPR-Cas9 for genome engineering. Cell. 2014; 157: 1262-1278.

47. Mali P, Yang L, Esvelt KM, Aach J, Guell M, DiCarlo JE. et al. RNA-guided human genome engineering via Cas9. Science. 2013; 339: 823-826.

48. Hu J, Lei Y, Wong WK, Liu S, Lee KC, He X. et al. Direct activation of human and mouse Oct4 genes using engineered TALE and Cas9 transcription factors. Nucleic Acids Res. 2014; 42: 4375-4390.

49. Mosbach V, Poggi L, Viterbo D, Charpertier M, Richard G-F. TALEN-Induced double-strand break repair of CTG trinucleotide repeats. Cell Reports. 2018; 22: 2146-2159.

50. Hu J, Matsui M, Corey DR. Allele-selective inhibition of mutant huntingtin by peptide nucleic acid-peptide conjugates, locked nucleic acid and small interfering RNA. Ann NY Acad Sci. 2009; 1175: 24-31. 
51. Kiliszek A, Banaszak K, Dauter Z, Rypniewski W. The first crystal structures of RNA-PNA duplexes and PNA-PNA duplex containing mismatches - toward anti-sense therapy against TREDs. Nucleic Acid Res. 2016; 44: 1937-1943.

52. Rué L, Bañez-Coronel M, Creus-Muncunill J, Giralt A, Alcalá-Vida R, Mentxaka G, et al. Targeting CAG repeat RNAs reduces Huntington's disease phenotype independently of huntingtin levels. J Clin Invest. 2016; 126: 4319-30.

53. Li L, Shen X, Liu Z, Norrbom M, Prakash TP, O'Reilly D, et al. Activation of frataxin protein expression by antisense oligonucleotides targeting the mutant expanded repeat. Nucleic Acid Ther. 2018; 28: 23-33.

54. Corey DR. Nusinersen, an antisense oligonucleotide drug for spinal muscular atrophy. Nat Neurosci. 2017; 20: 497-9.

55. Polak U, Li Y, Butler JS, Napierala M. Alleviating GAA repeat induced transcriptional silencing of the Friedreich's ataxia gene during somatic cell reprogramming. Stem Cells Dev. 2016; 25: 1788-1800.

56. Hockly E, Richon VM, Woodman B, Smith DL, Zhou X, Rosa E, et al. Suberoylanilide hydroxamic acid, a histone deacetylase inhibitor, ameliorates motor deficits in a mouse model of Huntington's disease. P Natl Acad Sci USA. 2003; 100: 2041-2046.

57. Mielcarek M, Benn CL, Franklin SA, Smith DL, Woodman B, Marks PA, et al. SAHA decreases HDAC 2 and 4 levels in vivo and improves molecular phenotypes in the R6/2 mouse model of Huntington's disease. PLOS ONE. 2011; 6: e27746.

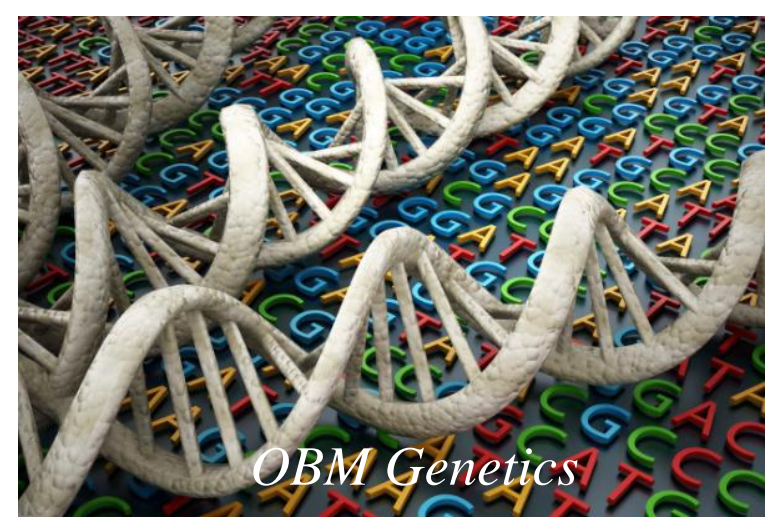

Enjoy $O B M$ Genetics by:

1. Submitting a manuscript

2. Joining in volunteer reviewer bank

3. Joining Editorial Board

4. Guest editing a special issue

For more details, please visit: http://www.lidsen.com/journals/genetics 